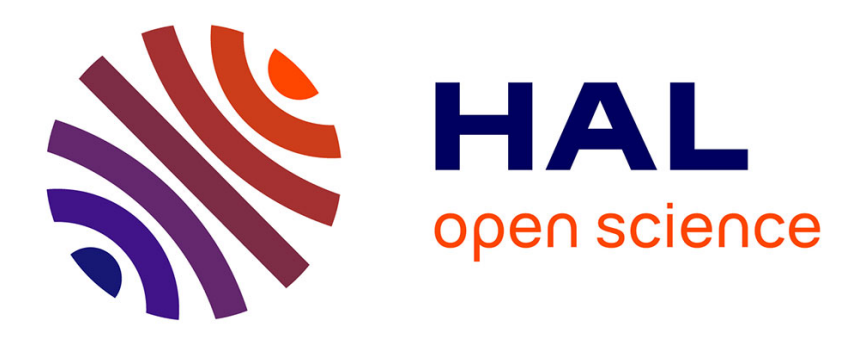

\title{
Détermination des positions des sources secondaires en contrôle acoustique actif
}

\author{
E. Benzaria, V. Martin
}

\section{To cite this version:}

E. Benzaria, V. Martin. Détermination des positions des sources secondaires en contrôle acoustique actif. Journal de Physique IV Proceedings, 1994, 04 (C5), pp.C5-191-C5-194. 10.1051/jp4:1994536 . jpa-00253031

\section{HAL Id: jpa-00253031 https://hal.science/jpa-00253031}

Submitted on 1 Jan 1994

HAL is a multi-disciplinary open access archive for the deposit and dissemination of scientific research documents, whether they are published or not. The documents may come from teaching and research institutions in France or abroad, or from public or private research centers.
L'archive ouverte pluridisciplinaire HAL, est destinée au dépôt et à la diffusion de documents scientifiques de niveau recherche, publiés ou non, émanant des établissements d'enseignement et de recherche français ou étrangers, des laboratoires publics ou privés. 


\title{
Détermination des positions des sources secondaires en contrôle acoustique actif
}

\author{
E. BENZARIA et V. MARTIN
}

Laboratoire de Mécanique et d'Acoustique, CNRS, 13402 Marseille cedex 20, France

\begin{abstract}
In the field of active noise control, the quality of the results achievable is highly dependent on the secondary source locations. Two strategies to determine these positions are investigated: the first one selects the sources among many and the second one consists in finding the configuration of sources which minimise the acoustic level, requiring a method to interpolate the transfer functions. A strategy which combinate selection and minimisation is proposed and applied to a numerical vibro-acoustic modelling of aircraft cabin, leading to an improvement of the results in comparison with those achieved previously.
\end{abstract}

\section{1- POSITION DU PROBLEME}

L'efficacité d'un système d'absorption acoustique active est grandement tributaire des positions des sources secondaires. Celles-ci peuvent être déterminées de manière discrète par sélection de positions dę sources prédéterminées [1,2], ou de manière continue dans l'espace, par la minimisation du niveau acoustique résiduel via une méthode d'interpolation des fonctions de transfert [3]. Après avoir appréhendé cette méthode pour 1 ddl [4], l'avoir appliquée sur un modèle numérique d'avion [5], on propose aujourd'hui sur le même modèle numérique l'outil $2 \mathrm{ddl}$ écrit en coordonnées cylindrique avec contrainte sur le domaine des sources secondaires. Du fait de la non convexité de la fonctionnelle du niveau résiduel en fonction de la position des sources, la qualité de la minimisation dépend grandement de la configuration initiale choisie. Attendu qu'il n'est pas concevable de tester toutes les configurations associées à un nombre de sources donné, une stratégie d'optimisation associant sélection et minimisation est proposée.

\section{2- FONDEMENTS DE LA MÉTHODE D'INTERPOLATION}

Soient NA sources secondaires de positions $s=\left(s_{k}\right)$, alimentées par les tensions complexes $\mathbf{a}=\left(\mathrm{a}_{\mathrm{k}}\right)$ dans le but d'atténuer un champ primaire harmonique po dans une zone de l'espace controlée par NM capteurs d'erreurs $\mathbf{m}=\left(\mathrm{m}_{\mathrm{i}}\right)$. Si $\mathbf{G}=\left(\mathrm{gi}_{\mathrm{i}, \mathrm{k}}\right)$ est la matrice des fonctions de transfert entre les $\mathrm{m}_{\mathrm{i}}$ et les $\mathrm{s}_{\mathrm{k}}$, le niveau acoustique résiduel dans cette zone de l'espace s'écrit:

$$
\begin{gathered}
J(\mathbf{a}, \mathbf{S}, \mathbf{M})=1 / 2\left[\mathbf{G} \cdot \mathbf{a}+\mathbf{p}_{\mathbf{0}}\right]^{*}\left[\mathbf{G} \cdot \mathbf{a}+\mathbf{p}_{\mathbf{0}}\right] \\
\text { a vérifiant l'expression: } \frac{\partial J}{\partial \mathbf{a}}(\mathbf{a}, \mathbf{s}, \mathbf{m})=0 \Leftrightarrow \mathbf{a}=-\left[\mathbf{G}^{*} \cdot \mathbf{G}\right]^{-1} \cdot \mathbf{G}^{*} \cdot \mathbf{p}_{\mathbf{0}}
\end{gathered}
$$

La minimisation de $\mathrm{J}(\mathrm{s})$ via un algorithme de gradient conjugé [6] nécessite la connaissance de $\mathrm{J}$ ainsi que du gradient de $\mathrm{J}$, et par suite de $\mathbf{G}$ et ses dérivées partielles premières par rapport à chaque source $S_{k}$, quelle que soit la configuration de sources. Pour ce faire, à la suite de [3], une méthode d'interpolation $2 \mathrm{D}$ a été mise en oeuvre. 


\section{2-1 Méthode d'interpolation 2D cylindrique.}

$\mathrm{Si} \mathrm{g}_{\mathrm{i}, \mathrm{j}}$ sont des fonctions de transfert connues entre un microphone $\mathrm{m}_{\mathrm{i}}$ quelconque et les points $\mathrm{e}_{\mathrm{j}}$ $(j=1,2,3)$, la fonction de transfert $g$ et ses dérivées premières partielles en un point $\mathbf{s}_{\mathbf{k}}$ intermédiaire des points $\mathrm{e}_{\mathrm{j}}$ sont interpolées en considérant le Développement de Taylor à l'ordre 2 de chaque $\mathrm{g}_{\mathrm{i}, \mathrm{j}}$. Dans les applications futures, les échantillons placés sur un cylindre, seront repérés par les variables $(\mathrm{r}, \theta, \mathrm{z})$. L'écriture rigoureuse du D.T en coordonnées cylindriques fait apparaître des coefficients dans lesquels interviennent $r, \cos (\theta), \sin (\theta)$. Si le rayon $r$ du cylindre est de l'ordre de 1 et les variations entre $\theta e_{j}$ et $\theta s_{k}$ sont petites, l'action de $r$ sur la distance entre $e_{j}$ et $s_{k}$ est négligeable et les variables $\theta$ et $z$ peuvent être directement identifiées avec les variables $\mathrm{y}, \mathrm{z}$ du développement de Taylor en coordonnées cartésiennes.

$$
\begin{aligned}
& g_{i, j}^{e}=\left\langle g_{i, k} \frac{\partial g_{i, k}}{\partial \theta_{k}} \frac{\partial g_{i, k}}{\partial z_{k}} \frac{\partial^{2} g_{i, k}}{\partial \theta_{k}{ }^{2}} \frac{\partial^{2} g_{i, k}}{\partial \theta \partial z_{k}} \frac{\partial^{2} g_{i, k}}{\partial z_{k}{ }^{2}}\right) \cdot\left[\begin{array}{c}
1 \\
\left(\delta \theta_{j-k}\right) \\
\left(\delta z_{j-k}\right) \\
\frac{1}{2}\left(\delta \theta_{j-k}\right)^{2} \\
\left(\delta \theta_{j-k}\right) \cdot\left(\delta z_{j-k}\right) \\
\frac{1}{2}\left(\delta z_{j-k}\right)^{2}
\end{array}\right\}+R_{j, k}=C_{i, k}^{T} \cdot U_{j, k}+R_{j, k} \\
& \text { avec } \delta \theta_{j-k}=\left(\theta e_{j}-\theta s_{k}\right), \delta z_{j-k}=\left(z e_{j}-z s_{k}\right), g_{i, k}=g\left(m_{i}, s_{k}\right)
\end{aligned}
$$

La colonne inconnue $C_{i, k}$ est alors déterminée en minimisant la somme des carrés pondérés par $W_{j, k}$ des restes $R_{j, k}(j=1, \ldots, 9$ par exemple) des développements de Taylor considérés aux 9 points d'échantillonnage (support de $\mathrm{W}_{\mathrm{j}, \mathrm{k}}$ ) les plus proches du points d'estimation.

$$
\begin{aligned}
& \min _{\mathbf{C}_{i, k}}\left(\sum_{j=1}^{g} W_{j, k} \cdot\left(R_{j, k}\right)^{2}\right)=\min _{C_{i, k}}\left(\sum_{j=1}^{9} W_{j, k} \cdot\left(g e_{i, j}-C_{i, k}^{T} \cdot U_{j, k}\right)^{2}\right) \\
& \text { avec } w\left(e_{j}, s_{k}\right)=10^{-p\left(\frac{d\left(e_{j}, s_{k}\right)}{d \max }\right)^{2}} \text { si } d\left(e_{j}, s_{k}\right)>d \max
\end{aligned}
$$

On résoud finalement le système:

$$
\mathbf{C}_{\mathrm{i}, \mathrm{k}}=\left[\sum_{\mathrm{j}=1}^{g} \mathrm{~W}_{\mathrm{j}, \mathrm{k}} \cdot \mathbf{U}_{\mathrm{j}, \mathrm{k}} \cdot \mathbf{U}_{\mathrm{j}, \mathrm{k}}^{\mathrm{T}}\right]^{-1} \cdot \sum_{\mathrm{j}=1}^{g} \mathrm{~W}_{\mathrm{j}, \mathrm{k}} \cdot \mathrm{ge}_{\mathrm{i}, \mathrm{j}} \cdot \mathbf{U}_{\mathrm{j}, \mathrm{k}}
$$

Comme la méthode des moindres carrés s'accompagne d'un plus grand nombre d'échantillons que d'inconnues, $\mathrm{W}_{\mathrm{j}, \mathrm{k}}$ minimise la contribution des échantillons les plus éloignés du point d'estimation via la pente $p$ et la valeur de dmax:

- la pente $\mathrm{p}$ définit un poid associé à chaque échantillon. Elle est choisie de manière à accentuer la contribution de l'échantillon le plus proche de $s_{\mathbf{k}}$ par rapport aux autres échantillons du support, dont l'influence sur $\mathrm{g}_{\mathrm{ik}}$, certe moindre, est indispensable au bon conditionnement de (6);

- dmax fixe la distance maximum entre $s_{k}$ et $e_{j}$ pour laquelle l'échantillon est pris en compte. Quand $s_{\mathrm{k}}$ s'approche des frontières du domaine échantillonné, le support de $\mathrm{W}_{\mathrm{j}, \mathrm{k}}$ est inévitablement tronqué. De plus, en présence d'un échantillonnage irrégulier, la valeur de dmax adaptée à l'èchantillonnage ne le serait qu'au détriment de la continuité des estimateurs. Ainsi, dmax a été fixé pour que au moins 8 échantillons soient pris en compte dans la minimisation, quelle que soit la position de $\mathrm{s}_{\mathrm{k}}$ sur le domaine.

\section{2-2 Application sur un exemple académique cylindrique}

Soit une source primaire située en $(x, y, z)=(0,3 \mathrm{~m}, 0)$ (fig 1$)$ et 77 microphones d'erreur dans le plan $\mathrm{y}=0.2 \mathrm{~m}$ limité par un cylindre immatériel sur lequel on a les valeurs des fonctions de transfert en champ indéfini (fonction de Green 3D) aux 99 points échantillons ej $=\left(\theta e_{j}, z_{j}\right)\left(9\right.$ valeurs de $\theta e_{j}$ sur le contour du cylindre dans chacune des 11 sections $\left.\mathrm{z}=\mathrm{ze}_{\mathrm{j}}\right)$. Une unique source secondaire $\mathrm{s}=(\theta, \mathrm{z})$ parcourt le cylindre et à partir des valeurs de $\mathrm{g}, \partial \mathrm{g} / \partial \theta_{\mathrm{S}}, \partial \mathrm{g} / \partial \mathrm{z}_{\mathrm{S}}$ obtenues via (6), les graphes des valeurs de $\mathrm{J}, \partial \mathrm{J} / \partial \theta_{\mathrm{S}}, \partial \mathrm{J} / \partial \mathrm{z}_{\mathrm{S}}$ au niveau de chaque microphone sont comparés aux valeurs exactes (fig 2). La qualité de l'interpolation est confirmée. 


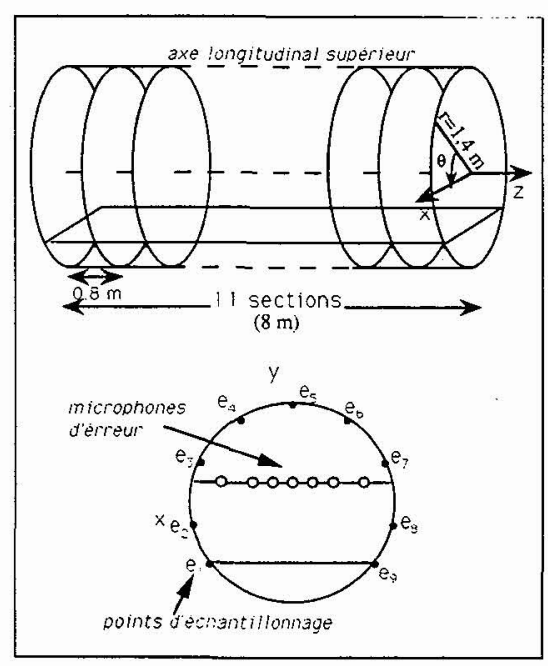

Fig 1: Configuration géométrique des modèles académique et numérique
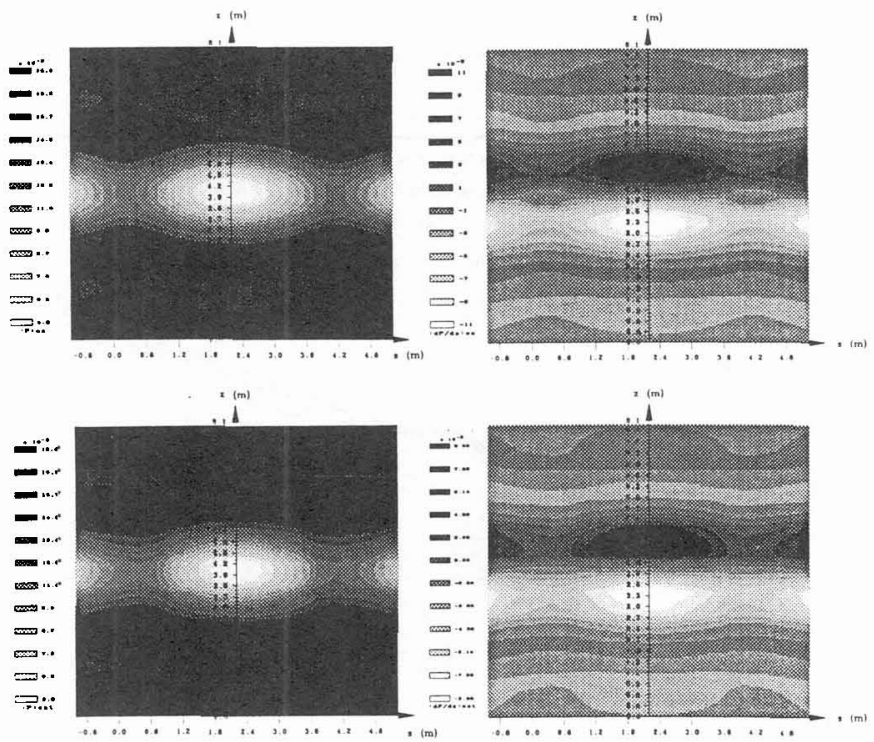

Fig 2: Cartes des pressions résiduelles (gauche) et de leurs dérivées par rapport à $\mathrm{z}$ (droite), valeurs exactes (haut) estimées (bas)

\section{STRATEGIE D'OPTIMISATION}

\section{3-1 Positions initiales et stratégies d'optimisation}

J étant non convexe, la qualité des résultats obtenus dépendra du choix de la configuration initiale de sources à partir de laquelle l'algorithme de gradient est implémenté. Pour ce faire [1] détermine cette configuration initiale à partir d'un grand nombre de sources placées en lieux des échantillons et en éliminant successivement celle de moindre commande (hypothèse de champ diffus). La stratégie qui consiste à faire la même sélection sur des sources de positions optimisées permet d'améliorer encore les résultats [5]. Cependant, ce mode de sélection présente les inconvénients suivants:

- le rôle de chacune des sources est d'autant plus important que sa position est optimisée. Celle de moindre commande n'est peut être pas la moins efficace;

- procéder par élimination restreint le nombre de configurations testées à celui des sources encore présentes. Rien ne dit qu'une source éliminée dans une configuration ne soit pas indispensable ultérieurement.

Pour palier à ces inconvenients, la stratégie d'optimisation de la fonctionnelle $\mathrm{J}$ a été la suivante:

- la sélection se fait par ajout d'une source choisie parmi les échantillons du modèle. L'optimisation ne débute plus à partir d'un grand nombre de sources, mais à partir d'une seule source dont la position est initialement optimisée;

- les sources sont sélectionnée selon un critère d'efficacité et non plus de commande: après minimisation de $\mathbf{J}$ en fonction des positions des sources, la source sélectionnée est celle qui donne la plus petite valeur de J. Une position de source déjà sélectionnée peut donc l'être à nouveau si par la minimisation de $\mathrm{J}$, la source a depuis changé de position;

- en outre, les sources sont contraintes à rester dans le domaine échantillonné.

\section{3- 2 Application sur un modèle numérique de cabine d'avion}

Le cylindre immatériel de la fig 1 est maintenant remplacé par un fuselage qui sépare l'excitation du champ primaire observé en 99 microphones dans le plan x0z représentant celui de la tête des passagers. Les échantillons situés aux noeuds du maillage de la structure traitée par éléments finis (face interne) sont irrégulièrement espacés selon $\theta$. Bien que l'interpolation utilise les 99 échantillons, la sélection, par 
cohérence avec le passé, ne se fait que sur 55 échantillons. Les résultats de l'optimisation sont comparés aux résultats obtenus sur le même problème avec les stratégies jusqu'alors utilisées.

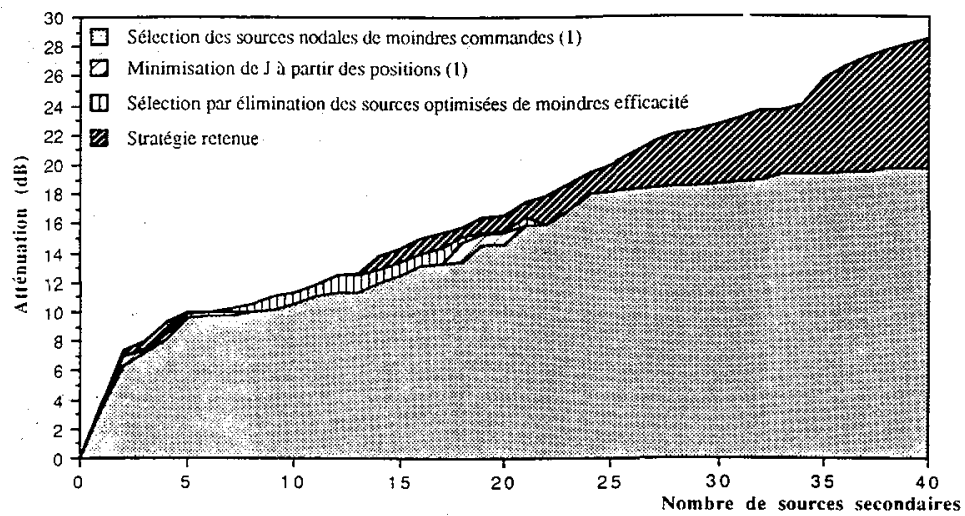

Fig 3: Atténuations obtenues par les diverses stratégies

Le gain en atténuation est visible à partir de 13 sources et croît fortement lorsque leur nombre augmente. La force de la présente stratégie repose sur le fait que des noeuds qui ont déjà été sélectionnés peuvent l'être à nouveau, permettant l'accroîssement de la densité de sources dans des région où elles sont éfficaces au détriment d'autres où elles le sont moins. Ces zones d'attraction avaient déjà été mise en évidence dans [5].

\section{4- ConClusion}

Disposant initialement de la courbe donnant l'atténuation relatives aux sources nodales sélectionnées et compte tenu que l'optimisation de la position des sources secondaires est un objectif que l'on ne peut atteindre à coup sûr du fait de la trés forte non convexité de la fonctionnelle, l'utilisation des outils présentés a permis d'améliorer sensiblement les résultats initiaux.

\section{RÉFÉRENCES}

[1] V. MARTIN, B. PESEUX, Ph. VIGNASSA A parattre dans le Journal of Sound and Vibration en 1994. Numerical vibroacoustic modelling of aircraft for the active acoustic control of interior noise.

[2] S. D. SNYDER, H. HANSEN: Journal of Sound and Vibration, (1991) 148(3), 537-542. Using multiple regressions to optimize active noise control system design.

[3] B. NAYROLES G. TOUZOT, P.VILLON A paraitre dans le Journal of Sound and Vibration en 1993. Using diffuse approximation for optimising the location of antisound sources.

[4] V. MARTIN, E. BENZARIA april 1993 Proceeding of 7th International Meeting on Low Frequency Noise \& Vibration, Edinburgh. Optimisation of secondary source locations (harmonic linear range).

[5] E. BENZARIA, V.MARTIN A paraitre dans le Journal of Sound and Vibration en 1994. Secondary source locations in active noise control: selection or optimisation?

[6] R. FLETCHER, C. M. REEVES 1964: Computer Journal 7. Function minimization by conjugate gradients. 